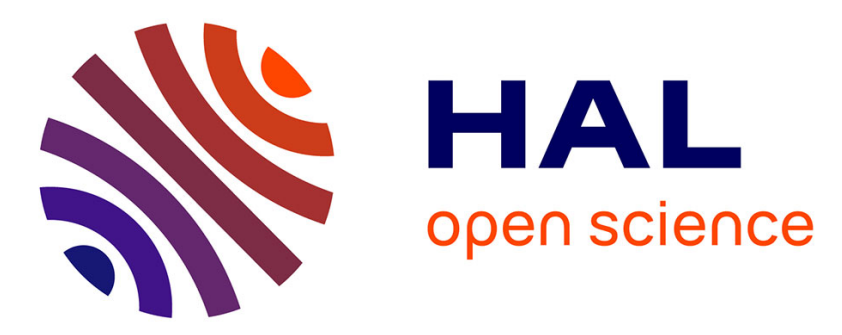

\title{
Time Integration and Assessment of a Model for Shape Memory Alloys Considering Multiaxial Nonproportional Loading Cases
}

Wael Zaki, Xiaojun Gu, Weihong Zhang, Claire Morin, Ziad Moumni

\section{- To cite this version:}

Wael Zaki, Xiaojun Gu, Weihong Zhang, Claire Morin, Ziad Moumni. Time Integration and Assessment of a Model for Shape Memory Alloys Considering Multiaxial Nonproportional Loading Cases. ASME 2014 Conference on Smart Materials, Adaptive Structures and Intelligent Systems, Sep 2014, Newport, United States. pp. V001T03A024; (5 p.), 2014, 978-0-7918-4614-8. 10.1115/SMASIS20147599 . emse-01108477

\section{HAL Id: emse-01108477 \\ https://hal-emse.ccsd.cnrs.fr/emse-01108477}

Submitted on 23 Jan 2015

HAL is a multi-disciplinary open access archive for the deposit and dissemination of scientific research documents, whether they are published or not. The documents may come from teaching and research institutions in France or abroad, or from public or private research centers.
L'archive ouverte pluridisciplinaire HAL, est destinée au dépôt et à la diffusion de documents scientifiques de niveau recherche, publiés ou non, émanant des établissements d'enseignement et de recherche français ou étrangers, des laboratoires publics ou privés. 


\title{
TIME INTEGRATION AND ASSESSMENT OF A MODEL FOR SHAPE MEMORY ALLOYS CONSIDERING MULTIAXIAL NONPROPORTIONAL LOADING CASES
}

\author{
Wael Zaki \\ Khalifa University \\ Xiojun Gu \\ 127788 Abu Dhabi \\ UAE \\ Northwestern Polytechnical University \\ 127 Youyi West Rd, Xi'an, Shaanxi 710072 \\ China \\ Claire Morin \\ École des Mines de Saint-Étienne \\ 158, cours Fauriel, 42023 Saint-Étienne \\ France \\ Ziad Moumni \\ ENSTA-ParisTech \\ 91762 Palaiseau Cedex \\ France \\ Weihong Zhang \\ Northwestern Polytechnical University \\ 127 Youyi West Rd, Xi'an, Shaanxi 710072 \\ China
}

\begin{abstract}
The paper presents a numerical implementation of the ZM model for shape memory alloys that fully accounts for non-proportional loading and its influence on martensite reorientation and phase transformation. Derivation of the time-discrete implicit integration algorithm is provided. The algorithm is used for finite element simulations using Abaqus, in which the model is implemented by means of a user material subroutine. The simulations are shown to agree with experimental and numerical simulation data taken from the literature.
\end{abstract}

\section{INTRODUCTION}

Shape memory alloys (SMAs) are capable of undergoing severe inelastic deformation that can be recovered by heating. Alternatively, beyond a certain temperature, the recovery of inelastic deformation can be accomplished by removal of the load to which the SMA is subjected. Such behavior is known as "superelasticity" because it holds phenomenological analogy with conventional elasticity, even though the underlying process is dissipative and gives rise to a hysteresis loop in the SMA stress-strain curve.
The present work focuses on proper numerical integration of the Zaki-Moumni model for SMAs [8, 17, 18] subjected to complex multiaxial loading in the superelastic range. The inelastic deformation of the material is accomplished in this case by means of two distinct physical processes: a solid-solid phase transformation from a single-variant parent phase called austenite to a multivariant product phase called martensite, and a reorientation of martensite by the formation of preferred variants at the expense of others as a result of changes in the magnitude and orientation of the stress experienced by the martensite. Other aspects of SMA behavior such as tensilecompressive asymmetry [14, 20], SMA dynamics [10], slip plasticity [19] and thermomechanical coupling [5] including cyclic effects $[6,7,9]$ are not considered here. The time integration of the Zaki-Moumni model for martensitic SMAs is addressed in $[15,16]$.

In the literature, several models for SMAs were proposed that account for multiaxial loading to various degrees of success. [4] developed a model for SMAs that was validated against experimental data obtained earlier by the same research group for samples subjected to nonproportional biaxial loading. [11] derived a phenomenological model where the state equations are derived from thermo- 
dynamic potentials in accordance with the principles of thermodynamics. The model was validated against experimental data obtained by [12]. Other models were also presented in [13], [2], and more recently in [1]. The latter uses the Fischer-Burgmeister functions to substitute the Kuhn-Tucker optimality conditions with nonlinear equalities in order to avoid the need for iterative detection of active loading surfaces.

Simulation results using the ZM model for SMAs are compared in this paper to some of those reported in the above references. The model is further validated using experimental data taken from the literature.

\section{ANALYTICAL DERIVATIONS}

Only key constitutive relations are listed here for conciseness. For details on the analytical derivation procedure for the model the reader is referred to $[17,18]$. The following state variables are considered: the volume fraction of martensite $z$, the local inelastic strain in the martensite phase due to reorientation $\boldsymbol{\varepsilon}^{\text {ori }}$, as well as the conventional total strain $\boldsymbol{\varepsilon}$ and temperature $T$. Following standard convention, tensors are represented with bold characters.

The ZM model uses the framework of generalized standard materials in deriving the constitutive relations for SMAs. The state equations are derived from the expression of a Helmholtz free energy density and include the following stress-strain relation:

$$
\boldsymbol{\sigma}=\boldsymbol{K}:\left(\boldsymbol{\varepsilon}-z \boldsymbol{\varepsilon}^{\text {ori }}\right),
$$

where $\boldsymbol{\sigma}$ is the stress tensor and $\boldsymbol{K}$ is the elastic stiffness tensor, which may depend on the volume fraction of martensite.

The above equation reflects that the total inelastic strain in a reference volume element of the SMA is resolved into the product of the amount of martensite that exists within that element and the amount of local inelastic strain experienced by the martensite due to reorientation of the variants.

Both $z$ and $\boldsymbol{\varepsilon}^{\text {ori }}$ are dissipative variables for which the evolution is governed by the loading functions $\mathcal{F}_{z}^{1}$ and $\mathcal{F}_{z}^{2}$ for forward $(\dot{z}>0)$ and reverse $(\dot{z}<0)$ phase transformations and $\mathcal{F}_{\text {ori }}$ for martensite reorientation. The loading functions depend on $\boldsymbol{\sigma}, z, \boldsymbol{\varepsilon}^{\text {ori }}$ and $T$. For the purpose of this manuscript, only isothermal processes are considered, temperature is therefore introduced only as a parameter.

The explicit expressions for the loading functions are the following:

$$
\begin{aligned}
\mathcal{F}_{z}^{1}= & F_{z}^{1}\left(\boldsymbol{\sigma}, z, \boldsymbol{\varepsilon}^{\text {ori }}\right) \\
= & \frac{1}{2}\left(\frac{3}{2} E l_{M A} \boldsymbol{s}: \boldsymbol{s}+P_{M A} \operatorname{tr}^{2}(\boldsymbol{\sigma})\right)-C(T) \\
& +\boldsymbol{s}: \boldsymbol{\varepsilon}^{\text {ori }}-(G+b) z-a(1-z) \\
& -\left[(\alpha-\beta) z+\frac{\beta}{2}\right]\left(\frac{2}{3} \boldsymbol{\varepsilon}^{\text {ori }}: \boldsymbol{\varepsilon}^{\text {ori }}\right),
\end{aligned}
$$

$$
\begin{aligned}
\mathcal{F}_{z}^{2}= & F_{z}^{2}\left(\boldsymbol{\sigma}, z, \boldsymbol{\varepsilon}^{\text {ori }}\right) \\
= & -\frac{1}{2}\left(\frac{3}{2} E l_{M A} \boldsymbol{s}: \boldsymbol{s}+P_{M A} \operatorname{tr}^{2}(\boldsymbol{\sigma})\right)+C(T) \\
& -\boldsymbol{s}: \boldsymbol{\varepsilon}^{\text {ori }}+(G-b) z-a(1-z) \\
& +\left[(\alpha-\beta) z+\frac{\beta}{2}\right]\left(\frac{2}{3} \boldsymbol{\varepsilon}^{\text {ori }}: \boldsymbol{\varepsilon}^{\text {ori }}\right), \\
& \mathcal{F}_{\text {ori }}=F_{\text {ori }}\left(\boldsymbol{\sigma}, z, \boldsymbol{\varepsilon}^{\text {ori }}\right)=X_{\mathrm{vm}}-z Y
\end{aligned}
$$

with

$$
\boldsymbol{X}=\boldsymbol{s}-\frac{2}{3 \varepsilon_{0}^{2}}\left(\boldsymbol{s}: \boldsymbol{\varepsilon}^{\text {ori }}\right) \boldsymbol{\varepsilon}^{\text {ori }}
$$

where $\boldsymbol{s}$ is the stress deviator, $\operatorname{tr}(\boldsymbol{\sigma})$ is the trace of the stress tensor, $X_{\mathrm{vm}}$ is the von Mises equivalent of the thermodynamic force $\boldsymbol{X}, C(T)$ is a linear function of temperature and $E l_{M A}, P_{M A}, \alpha, \beta, a, b, G$ are material parameters. The evolution of $\boldsymbol{\varepsilon}^{\text {ori }}$ is governed by the normality rule

$$
\dot{\boldsymbol{\varepsilon}}^{\text {ori }}=\eta \frac{\partial \mathcal{F}_{\text {ori }}}{\partial \boldsymbol{X}}=\frac{3}{2} \eta \frac{\boldsymbol{X}}{X_{v m}}=\eta \boldsymbol{N}
$$

where $\eta$ is an inelastic multiplier and $\boldsymbol{N}=\frac{3}{2} \frac{\boldsymbol{X}}{X_{v m}}$ is the direction of the orientation strain rate $\dot{\boldsymbol{\varepsilon}}^{\text {ori }}$ in strain space. The inelastic multiplier $\eta$ and the rate of phase transformation $\dot{z}$ are governed by standarrd Kuhn-Tucker conditions.

\section{ALGORITHMIC SETUP}

The problem to be solved is that of a SMA structure subjected to arbitrary mechanical loading over a time interval $[0, T]$. Following standard incremental solution procedure, the time interval is discretized into $N$ subintervals. Starting from a well-defined initial state, the time-discrete incremental problem consists in determining the values of the state variables everywhere in the structure for every load increment $n \in 1, N$ where $n=0$ corresponds to the initial state. Most finite element analysis software use a strain-controlled approach in which an increment of strain is first computed to satisfy the global equilibrium of the structure for a given load increment, the local constitutive equations are then used to compute the corresponding increments of stress and internal state variables. Local consistency with the constitutive equations is commonly enforced using a Newton-Raphson algorithm, which requires the derivation of a so-called Material Jacobian matrix that represents the rate of change of the increment of stress in terms of the increment of strain.

Assuming strain-controlled time-integration and using the symbol $\Delta$ to indicate time-discrete increments, the time-discrete equations for the SMA model used here are written as follows: 
1. Elastic predictor:

$$
\begin{aligned}
& \text { Set } k=0, \\
& \boldsymbol{\varepsilon}_{n+1}^{(k)}=\boldsymbol{\varepsilon}_{n}+\Delta \boldsymbol{\varepsilon} \\
& z_{n+1}^{(k)}=z_{n} \\
& \boldsymbol{\varepsilon}_{n+1}^{\text {ori }(k)}=\boldsymbol{\varepsilon}_{n}^{\text {ori }} \\
& \boldsymbol{\sigma}_{n+1}^{(k)}=\boldsymbol{K}_{n}:\left(\boldsymbol{\varepsilon}_{n+1}^{(k)}-z_{n+1}^{(k)} \boldsymbol{\varepsilon}_{n+1}^{\text {ori }(k)}\right)
\end{aligned}
$$

2. Consistency conditions:

(a) Loading functions

$$
\begin{aligned}
& \mathcal{F}_{z}^{1(k)}=F_{z}^{1}\left(\boldsymbol{\sigma}^{(k)}, z^{(k)}, \boldsymbol{\varepsilon}^{\text {ori }(k)}\right) \\
& \mathcal{F}_{z}^{2(k)}=F_{z}^{2}\left(\boldsymbol{\sigma}^{(k)}, z^{(k)}, \boldsymbol{\varepsilon}^{\text {ori }(k)}\right) \\
& \mathcal{F}_{\text {ori }}^{(k)}=F_{\text {ori }}\left(\boldsymbol{\sigma}^{(k)}, z^{(k)}, \boldsymbol{\varepsilon}^{\text {ori }(k)}\right)
\end{aligned}
$$

(b) Active loading set

If $z^{(k)}<1$ and $\mathcal{F}_{z}^{1(k)}>0$ then forward phase change,

If $z^{(k)}>0$ and $\mathcal{F}_{z}^{2(k)}>0$ then reverse phase change,

If $\mathcal{F}_{\text {ori }}^{(k)}>0$ then martensite reorientation.

(c) Increments of internal variables

If forward phase change and no martensite reorientation then solve $\mathcal{F}_{z}^{1(k+1)}=0$ for $\Delta z^{(k+1)}, \Delta \eta^{(k+1)}=0$,

If reverse phase change and no martensite reorientation, solve $\mathcal{F}_{z}^{2(k+1)}=0$ for $\Delta z^{(k+1)}, \Delta \eta^{(k+1)}=0$,

If martensite reorientation and no phase transformation then solve $\mathcal{F}_{\text {ori }}^{(k+1)}=0$ for $\Delta \eta^{(k+1)}, \Delta z^{(k+1)}=0$,

If forward phase change and martensite reorientation then solve the system $\left\{\mathcal{F}_{z}^{1(k+1)}=0, \mathcal{F}_{\text {ori }}^{(k+1)}=0\right\}$ for $\Delta z^{(k+1)}$ and $\Delta \eta^{(k+1)}$,

If reverse phase change and martensite reorientation then solve the system $\left\{\mathcal{F}_{z}^{2(k+1)}=0, \mathcal{F}_{\text {ori }}^{(k+1)}=0\right\}$ for $\Delta z^{(k+1)}$ and $\Delta \eta^{(k+1)}$.

(d) Positivity of multipliers

If forward phase change and $\Delta z^{(k+1)}<0$ then set $\Delta z^{(k+1)}=0$, forward phase change is inactive,

If reverse phase change and $-\Delta z^{(k+1)}<0$ then set $\Delta z^{(k+1)}=0$, reverse phase change is inactive,

If martensite reorientation and $\Delta \eta^{(k+1)}<0$ then set $\Delta \eta^{(k+1)}=0$, martensite reorientation is inactive. (e) Consistency with intrinsic constraints

If forward phase change and $z_{n}+\Delta z^{(k+1)}>$ 1 then $\Delta z^{(k+1)}=1-z_{n}$,

If forward phase change and $z_{n}+\Delta z^{(k+1)}<$ 0 then $\Delta z^{(k+1)}=-z_{n}$.

(f) Set $k=k+1$, update $\boldsymbol{\sigma}^{(k)}, z^{(k)}, \boldsymbol{\varepsilon}^{\text {ori }(k)}, \boldsymbol{N}^{(k)}$ and repeat steps $b$ to e until consistency with the loading conditions and the intrinsic constraints on $z$ and $\boldsymbol{\varepsilon}^{\text {ori }}$ is achieved.

3. Internal variables and stress update

$$
\begin{aligned}
& z_{n+1}=z_{n}+\Delta z, \\
& \boldsymbol{\varepsilon}_{n+1}^{\text {ori }}=\boldsymbol{\varepsilon}_{n}^{\text {ori }}+\Delta \eta \boldsymbol{N}_{n+1}, \\
& \boldsymbol{\sigma}_{n+1}^{\text {trial }}=\boldsymbol{K}_{n+1}:\left(\boldsymbol{\varepsilon}_{n+1}-z_{n+1} \boldsymbol{\varepsilon}_{n+1}^{\text {ori }}\right) .
\end{aligned}
$$

In the above procedure, the equations $\mathcal{F}=0$, where $\mathcal{F}=$ $F\left(\boldsymbol{\sigma}, z, \boldsymbol{\varepsilon}^{\text {ori }}\right)$ and $F$ is any of the loading functions, can be solved at iteration $k+1$ using a Newton-Raphson algorithm such that

$$
\begin{aligned}
\mathcal{F}^{(k+1)} & \approx \mathcal{F}^{(k)}+\mathcal{F}_{, \boldsymbol{\sigma}}^{(k)}: \Delta \boldsymbol{\sigma}^{(k+1)} \\
& +\mathcal{F}_{, z}^{(k)}: \Delta z^{(k+1)}+\mathcal{F}_{, \boldsymbol{\varepsilon}^{\text {ori }}}^{(k)}: \Delta \boldsymbol{\varepsilon}^{\text {ori }(k+1)}
\end{aligned}
$$

where a comma used in the subscript indicates differentiation with respect to the subsequent variable. Introducing the approximate normality rule

$$
\Delta \boldsymbol{\varepsilon}^{\text {ori }(k+1)} \approx \Delta \eta^{(k+1)} \boldsymbol{N}^{(k)}
$$

where the direction tensor $\boldsymbol{N}$ is approximated using its value at iteration $k$, the above leads to a linear algebraic equation with two unknowns $\Delta z^{(k+1)}$ and $\Delta \eta^{(k+1)}$. A system of two such equations is solved every time the increments of the internal variables are determined.

\section{NUMERICAL SIMULATION AND VALI- DATION}

The model is used to simulate the experiment reported by Bouvet et al. in [3] for a SMA tube subjected to a combination of tension and internal pressure. The parameters of the model are determined using the experimental curve in figure 1 and the simulated curve is shown on the same figure for comparison. The obtained parameters are listed in table 1. In this table, $E_{A}$ and $E_{M}$ and Young's moduli for austenite and martensite, $v$ is Poisson's coefficient for the SMA, $Y$ is the stress onset for martensite detwinning at low temperature, $\xi$ and $\kappa$ are parameters used to define the function $C(T)$, and $A_{f}^{0}$ is the austenite-finish transformation temperature at zero stress.

The loading to which the cylinder is subjected corresponds to the axial and hoop stresses reported in figure 2. The behavior of the material is reported in terms of hoop 


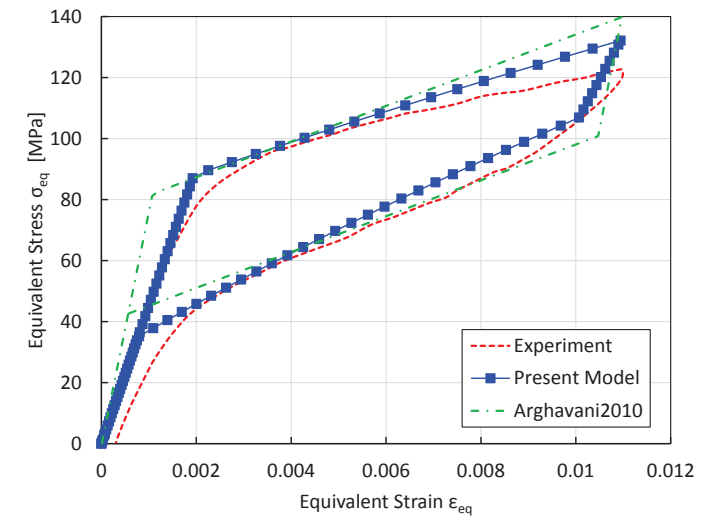

Fig. 1: Experimental and fitted stress-strain curves for the experiment of Bouvet et al. in [3].

\begin{tabular}{lccc}
\hline Parameter & Value & Parameter & Value \\
\hline$E_{A}$ & $82675 \mathrm{MPa}$ & $a$ & $4.79 \mathrm{MPa}$ \\
$E_{M}$ & $35000 \mathrm{MPa}$ & $b$ & $5.39 \mathrm{MPa}$ \\
$v$ & 0.4 & $\varepsilon_{0}$ & 0.038 \\
$Y$ & $30 \mathrm{MPa}$ & $G$ & $4.48 \mathrm{MPa}$ \\
$\alpha$ & $789.5 \mathrm{MPa}$ & $\beta$ & $3421 \mathrm{MPa}$ \\
$\xi$ & $0.19 \mathrm{MPa}$ & $\kappa$ & $2.32 \mathrm{MPa}$ \\
$A_{f}^{0}$ & $300 \mathrm{~K}$ & & \\
\hline
\end{tabular}

Table 1: Parameters used for simulating the experiment of Bouvet et al..

vs axial strain in figure 3 and in terms of the stress-strain curve in the axial and hoop directions in figures 4 and 5 . The simulation results are in good agreement with the experimental data for the first, second, and fourth loading steps. A marked deviation is observed however for the second loading step, in which the variation in hoop strain is significantly underestimated by the model. This may be explained by anisotropic material behavior in the axial and hoop directions that is not accounted for by the present model.

\section{CONCLUSION}

An integration procedure for a model for shape memory alloys was presented that accounts for complex nonproportional loading cases in the superelastic range. The numerical integration procedure for the model was presented, including the steps necessary for the detection of active loading sets and the enforcement of intrinsic and consistency constraints on the state variables. The approach used is analogous to classical multisurface plasticity. The model was successfully used to simulate experimental data taken from the literature for a SMA sample subjected to biaxial loading.

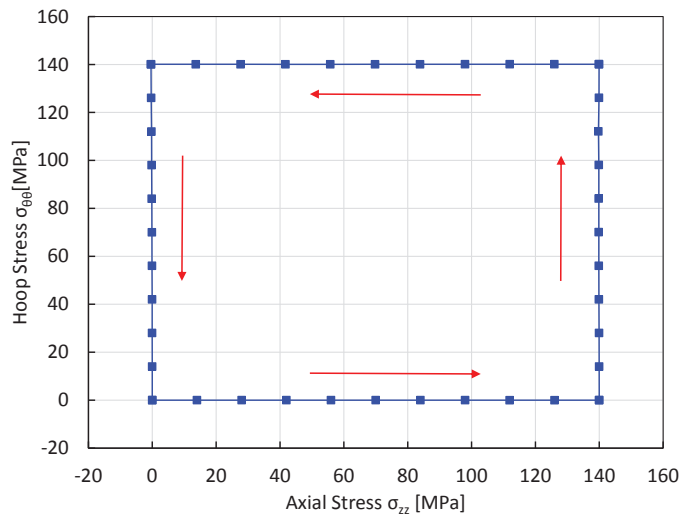

Fig. 2: Nonproportional loading considered for the simulation.

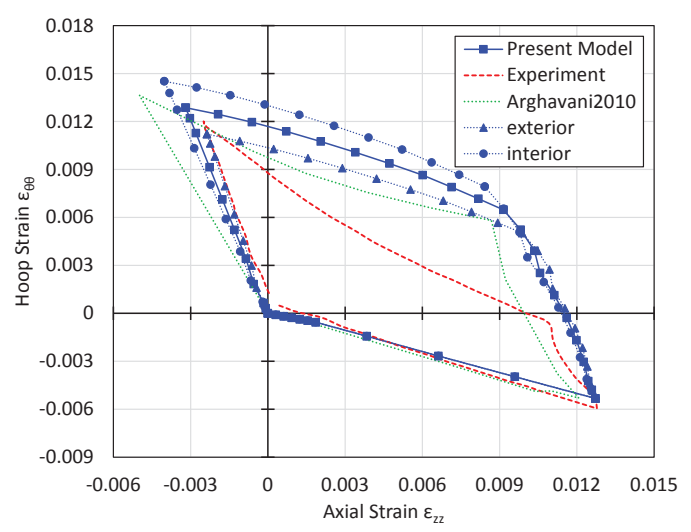

Fig. 3: Hoop vs axial strain in the SMA cylinder.

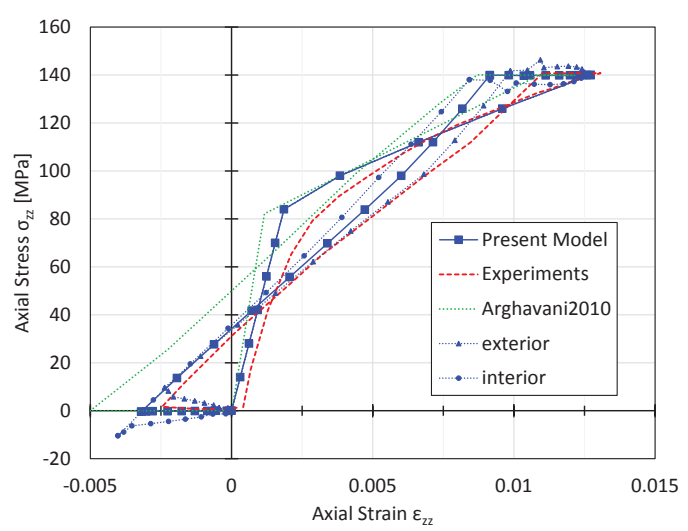

Fig. 4: Axial stress-strain response for the SMA cylinder.

\section{Acknowledgement}

The author would like to acknowledge the financial support of Khalifa University through KUIRF level 2 fund no. 21031. 


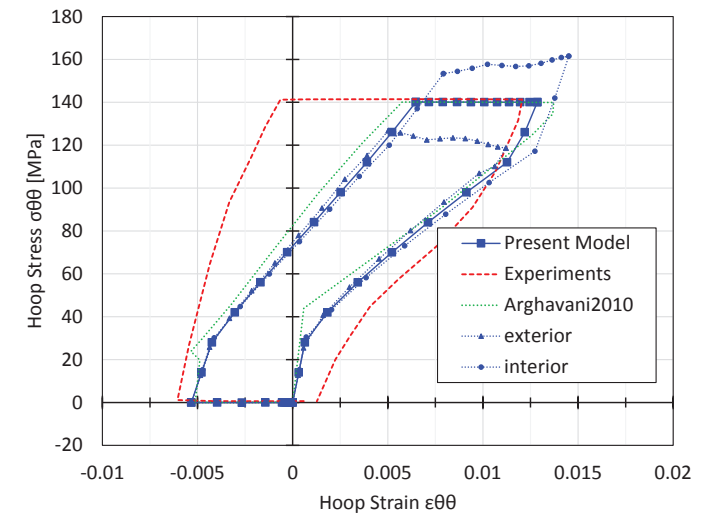

Fig. 5: Hoop stress-strain response for the SMA cylinder.

\section{REFERENCES}

[1] F. Auricchio, E. Bonetti, G. Scalet, and F. Ubertini, 2014. "Theoretical and numerical modeling of shape memory alloys accounting for multiple phase transformations and martensite reorientation". International Journal of Plasticity. ISSN 0749-6419.

[2] M. Bodaghi, A. R. Damanpack, M. M. Aghdam, and M. Shakeri, 2013. "A phenomenological SMA model for combined axial-torsional proportional/non-proportional loading conditions". Materials Science and Engineering: A, 587(0):1226.

[3] C. Bouvet, S. Calloch, and C. Lexcellent, 2002. "Mechanical Behavior of a Cu-Al-Be Shape Memory Alloy Under Multiaxial Proportional and Nonproportional Loadings". Journal of Engineering Materials and Technology, 124(2):112-124.

[4] C. Bouvet, S. Calloch, and C. Lexcellent, 2004. "A phenomenological model for pseudoelasticity of shape memory alloys under multiaxial proportional and nonproportional loadings". European Journal of Mechanics - A/Solids, 23(1):37-61.

[5] C. Morin, Z. Moumni, and W. Zaki, 2011. "A constitutive model for shape memory alloys accounting for thermomechanical coupling". International Journal of Plasticity, 27(5):748-767.

[6] C. Morin, Z. Moumni, and W. Zaki, 2011. "Thermomechanical coupling in shape memory alloys under cyclic loadings: Experimental analysis and constitutive modeling". International Journal of Plasticity, 27(12):1959-1980.

[7] C. Morin, Z. Moumni, and W. Zaki, 2011. "Direct numerical determination of the asymptotic cyclic behavior of pseudoelastic shape memory structures". Journal of Engineering Mechanics, 137(7):497 503.

[8] Z. Moumni, W. Zaki, and Q. S. Nguyen, 2008. "Theoretical and numerical modeling of solid-solid phase change: Application to the description of the thermomechanical behavior of shape memory alloys".
International Journal of Plasticity, 24(4):614-645.

[9] Z. Moumni, W. Zaki, and H. Maïtournam, 2009. "Cyclic behavior and energy approach to the fatigue of shape memory alloys". Journal of Mechanics of Materials and Structures, 4:395-411.

[10] M. Ould Moussa, Z. Moumni, O. Doaré, C. Touzé and W. Zaki, 2012. "Non-linear dynamic thermomechanical behaviour of shape memory alloys". Journal of Intelligent Material Systems and Structures, 23(14):1593-1611.

[11] M. Panico and L. C. Brinson, 2007. "A threedimensional phenomenological model for martensite reorientation in shape memory alloys". Journal of the Mechanics and Physics of Solids, 55(11):24912511.

[12] P. Sittner, Y. Hara, and M. Tokuda, 1995. "Experimental study on the thermoelastic martensitic transformation in shape memory alloy polycrystal induced by combined external forces". Metallurgical and Materials Transactions A, 26(11):2923-2935.

[13] A. P. Stebner and L. C. Brinson, 2013. "Explicit finite element implementation of an improved three dimensional constitutive model for shape memory alloys". Computer Methods in Applied Mechanics and Engineering, 257(0):17-35.

[14] W. Zaki, 2010. "An approach to modeling tensilecompressive asymmetry for martensitic shape memory alloys". Smart Materials and Structures.

[15] W. Zaki, 2012. "Time integration of a model for martensite detwinning and reorientation under nonproportional loading using Lagrange multipliers". International Journal of Solids and Structures, 49 (21):2951-2961.

[16] W. Zaki, 2012. "An efficient implementation for a model of martensite reorientation in martensitic shape memory alloys under multiaxial nonproportional loading". International Journal of Plasticity, 37:72-94.

[17] W. Zaki and Z. Moumni, 2007. "A three-dimensional model of the thermomechanical behavior of shape memory alloys". Journal of the Mechanics and Physics of Solids, 55(11):2455-2490.

[18] W. Zaki and Z. Moumni, 2007. "A 3D model of the cyclic thermomechanical behavior of shape memory alloys". Journal of the Mechanics and Physics of Solids, 55(11):2427-2454.

[19] W. Zaki, S. Zamfir, and Z. Moumni, 2010. “An extension of the ZM model for shape memory alloys accounting for plastic deformation". Mechanics of Materials, 42(3):266 - 274.

[20] W. Zaki, Z. Moumni, and C. Morin, 2011. "Modeling tensile-compressive asymmetry for superelastic shape memory alloys". Mechanics of Advanced Materials and Structures, 18(7):559-564. 